\title{
Multiloop Edgewise Arch-wire Technique for Skeletal Class III Openbite: A Case Report
}

\author{
Dr Dharma Laxmi Basukala \\ Orthodontist, Public Health Care Center, Bhaktapur, Nepal
}

Correspondence: Dr Dharma Laxmi Basukala; Email: dlbasukala@gmail.com

\section{ABSTRACT}

Skeletal Class III with openbite is one of the most difficult malocclusion to treat orthodontically. Generally, the morphological characteristics of this malocclusion are poor antero-posterior growth of the maxilla or excessive growth of the mandible with high angle. An 18-year-old male had Class III malocclusion with retrognathic maxilla and normal mandible with high angle. All third molars except on fourth quadrant were extracted to eliminate the posterior crowding. Multiloop Edgewise Arch-wire (MEAW) technique was used to upright the mesially inclined buccal teeth and to correct occlusal plane. In nine month time, anterior cross bite and open bite were corrected; normal overjet, overbite and Class I canine relation were achieved.

Keywords: Class III malocclusion, Multiloop Edgewise Arch-wire, openbite

\section{INTRODUCTION}

Skeletal Class III open bite is usually characterized by a high mandibular plane angle, obtuse gonial angle, overdeveloped mandible or underdeveloped maxilla, excessive vertical dimension of the upper molar, flattening of the occlusal plane, labial tipping of the maxillary anterior teeth, lingual tipping of the mandibular anterior teeth and a small cranial base angle. It may displace glenoid fossa anteriorly to cause a forward positioning of the mandible. These factors generally contribute to the development of skeletal malocclusion as well as facial deformities, and are believed to originate from genetic and/or environmental factors.'

The posterior discrepancy is an important etiological factor in the development of a skeletal Class III malocclusion because it affects occlusal plane. The occlusal plane is the most important component affecting the lower face vertically. The vertical position of posterior teeth in Class III malocclusion is not stable during growth and development. ${ }^{2-3}$ Continuous molar eruption occurs not only during growth of the facial structure but also during the post pubertal growth period. ${ }^{4-5}$

When the supra-eruption of molars occurs, several unfavorable changes take place. If the patient's growth potential is low the mandible may rotate backward. Consequently, an anterior open bite may be created.
However, if the patent has sufficient growth potential, vertical growth of condyle is stimulated, then the mandible rotates forward creating a skeletal Class III malocclusion. ${ }^{6}$

Genetics may not be the sole reason this type of Class III malocclusion develops; rather the continued eruption of second and third molar in a limited space may be the major contributing factor. A developing Class III malocclusion may be considered an effect of the posterior discrepancy or posterior crowding which is related to the insufficient eruption space caused by the insufficient anterior-posterior diameter due to an increased vertical growth of the maxilla. ${ }^{7}$

Here, we used MEAW technique developed by $\mathrm{Kim}^{8}$ to reconstruct the occlusal plane and correct the Class III malocclusion. This system typically includes the following changes:

1. The Posterior teeth were intruded and uprighted so that the occlusal plane could be reconstructed and the mandible repositioned posteriorly

2. Repositioning the mandible might prevent overgrowth of the condyle

3. The maxilla could be protracted downward and forward.

To eliminate the posterior discrepancy, the upper and lower third molars should be extracted prior to the onset of treatment. 

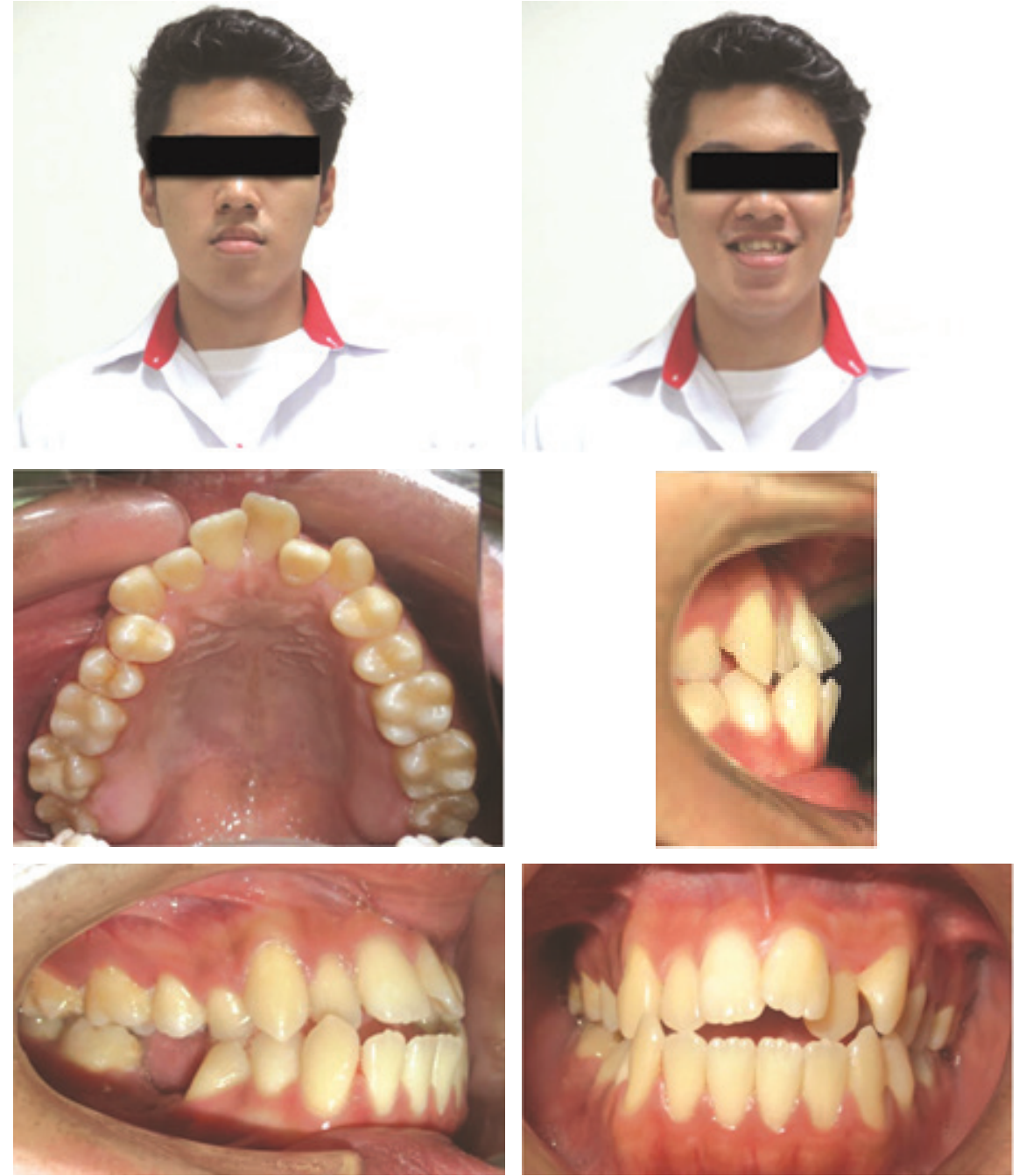

Figure 1: Pretreatment photographs
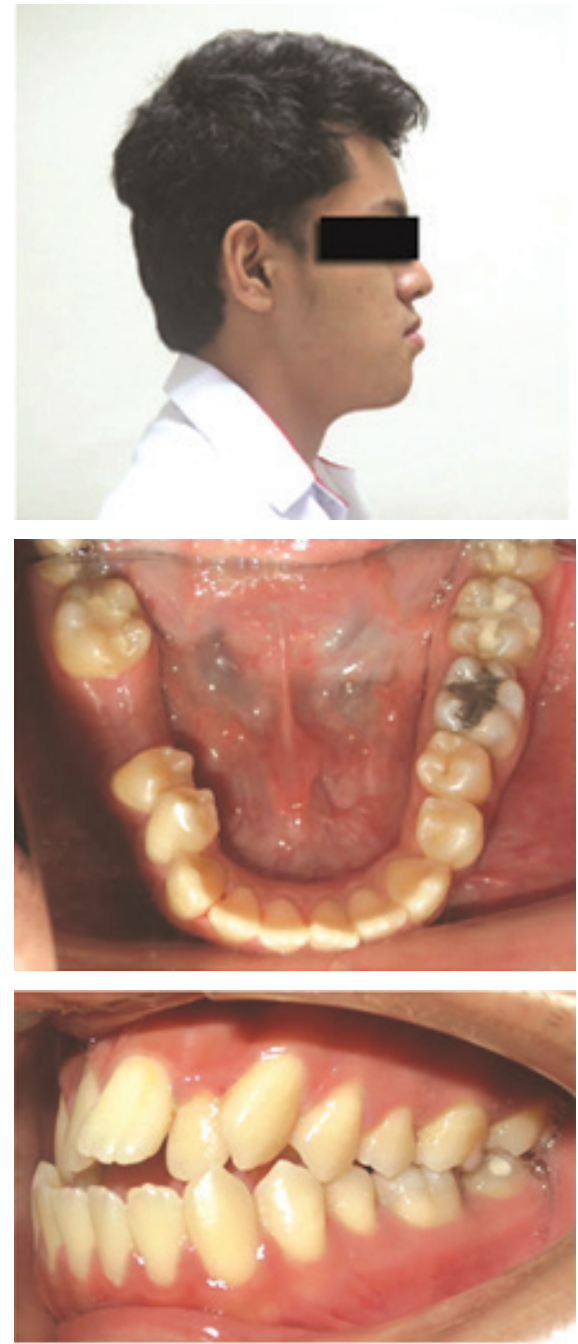

\section{CASE REPORT}

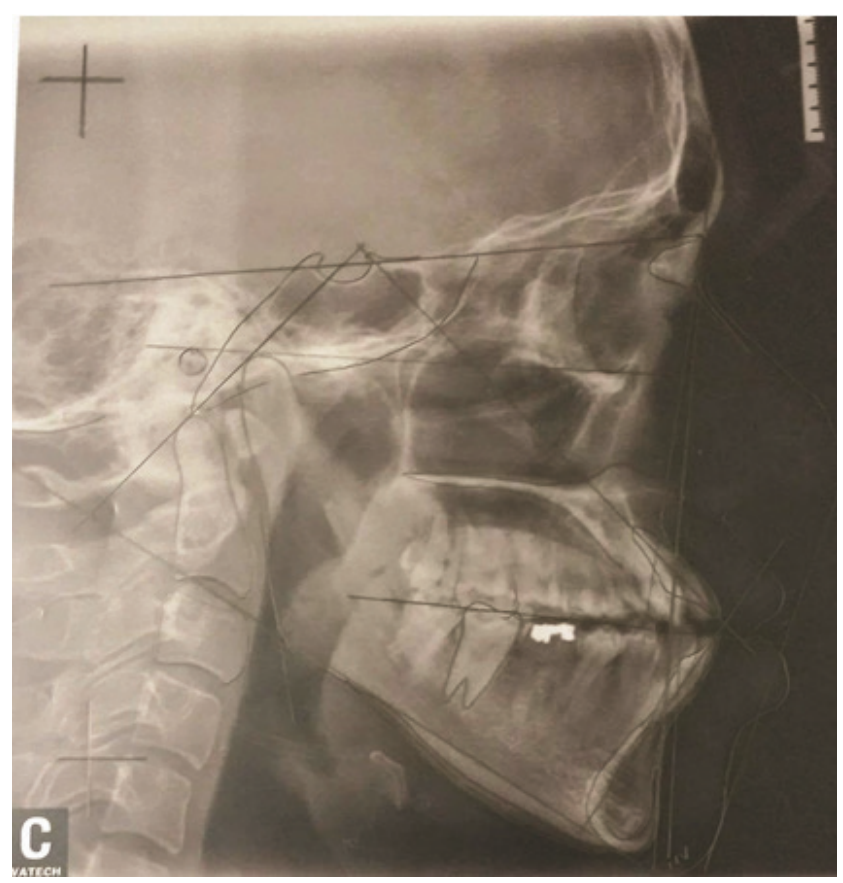

Figure 2: Pre-treatment cephalogram

An 18-year-old male patient came for orthodontic treatment with oblong facial profile and almost symmetrical face. Mandibular protrusion was observable. Intraoral examination revealed $V$-shaped upper arch with anterior crowding, anterior open bite, anterior cross bite, mandibular midline shift to right side by $2 \mathrm{~mm}$ and canine in Class III relation. Mandibular first right molar was extracted due to caries (Figure 1).

Cephalometric analysis showed skeletal Class III with retrognathic maxilla, normal mandible with high angle and anterior open bite (Figure 2).

The primary treatment objectives were to eliminate the posterior discrepancy, control the occlusal plane and simultaneously reposition the mandible distally. All third molars except on fourth quadrant (due to missing first molar) were extracted to eliminate the posterior crowding, to upright the mesially inclined buccal teeth and to change the occlusal plane. 

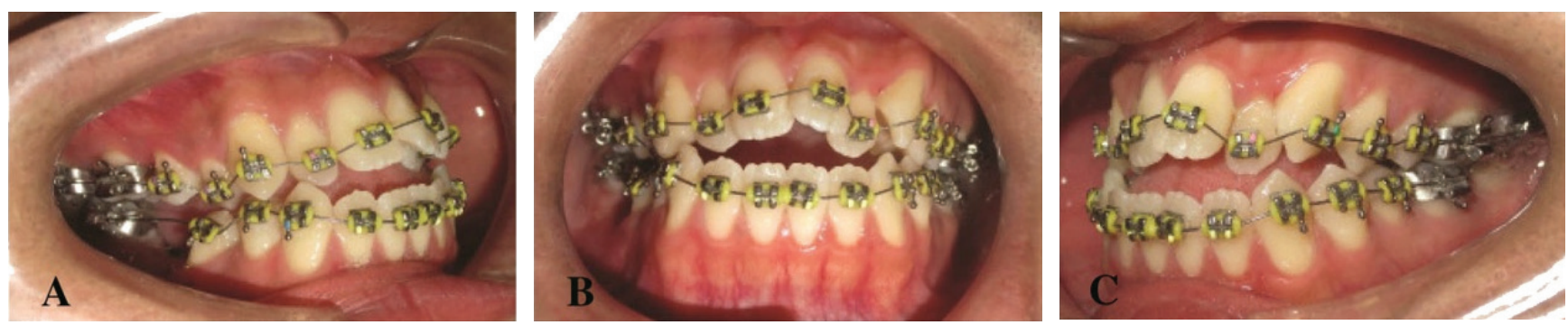

Figure 3: A,B and C - Leveling and Alignment-018 Edgewise Bracket system placed with 014 NiTi Archwire
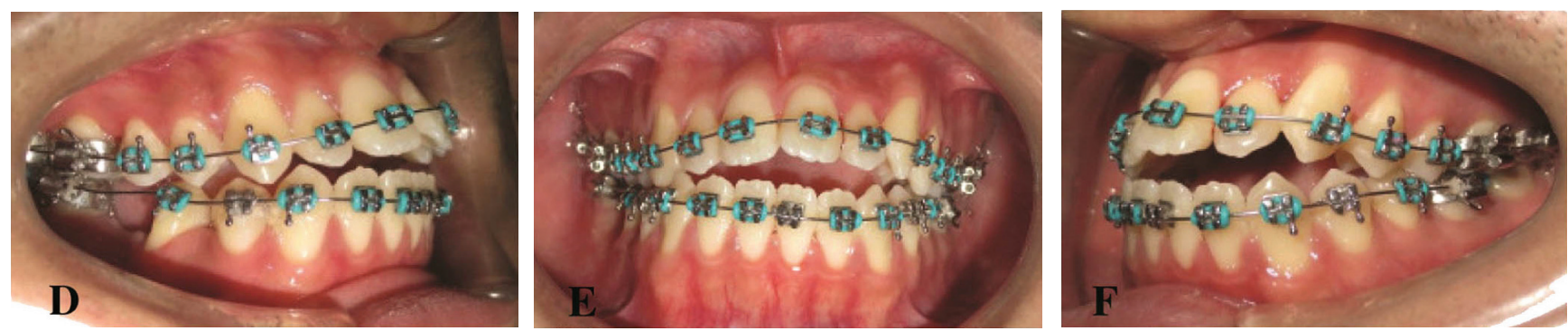

Figure 4: D,E and F - Leveling and Alignment Follow up $016 \mathrm{NiTi}$
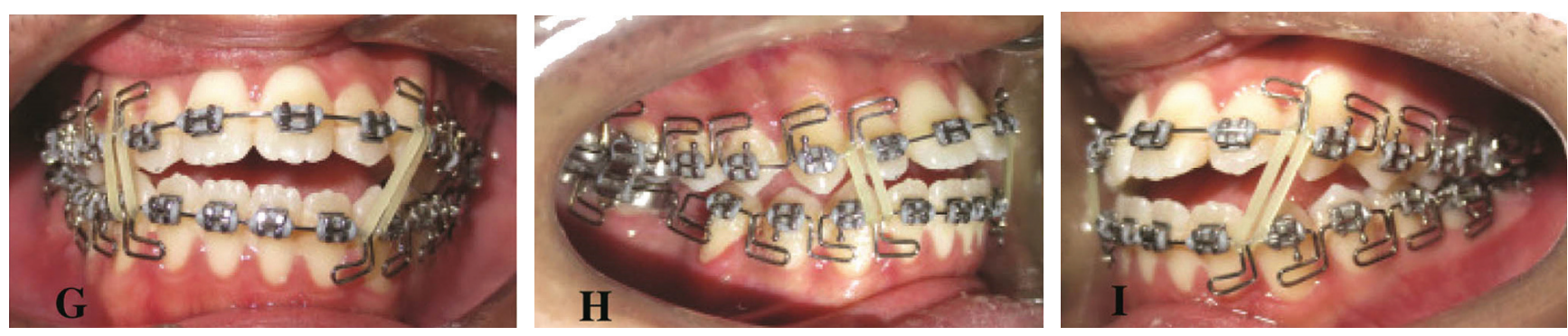

Figure 5: G,H and I - MEAW placement with Vertical Elastic (3/16 inch 6 oz)
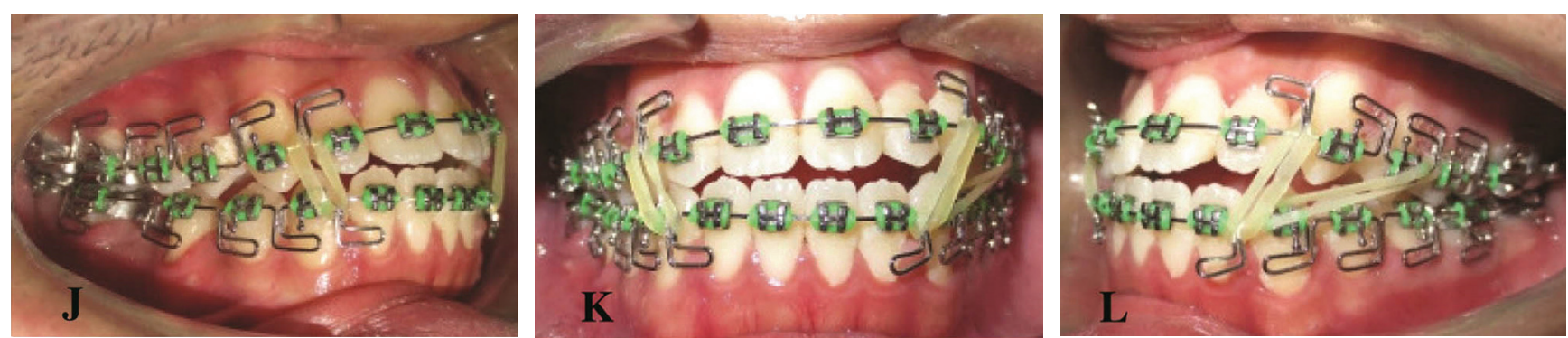

Figure 6: J,K and L - MEAW placement with Vertical and Class III Vector Elatic on left side
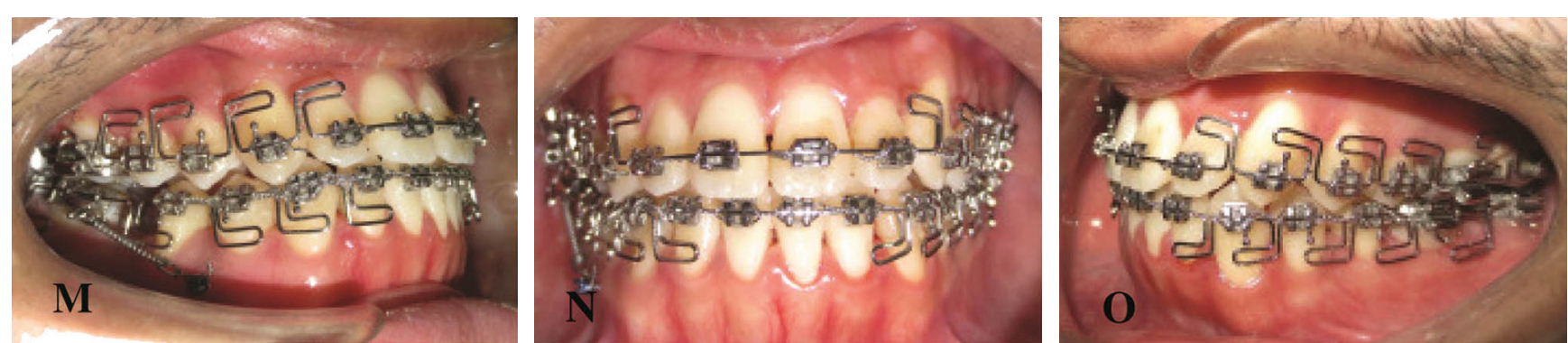

Figure 7: $M, N$ and $O$ - After 9 months of MEAW Therapy, open bite was corrected

Patient was banded and bonded with 0.018 slot edgewise bracket in both arches. Leveling and alignment was started with the use of $0.014 \mathrm{NiTi}, 0.016$ NiTi, followed by Multiloop Edgewise Arch-wire (MEAW) using 016X022 Blue Elgialloy in both arches in order to eliminate the molar interference. Alignment and intrusion was started through a progressive tip back bend activation from the premolar teeth to the molar areas using a vertical elastic (3/16 inch, 6oz) in the anterior and short Class III elastics (3/16 inch, 6 oz) on left side (Figure 3-7). 
Mandibular position was distalized due to the decrease of vertical dimension in the molar area. The anterior teeth overlap was improved by obtaining a physiologic vertical dimension. Then the tip back bends in the upper and lower molar area was removed and the MEAW was flattened for the protraction in fourth quadrant. An orthodontic mini implant (1.6/6 mm) was placed between 34 and 35 for molar protraction in fourth quadrant.

The case is in finishing stage and due for debonding in few months.

\section{DISCUSSION}

Skeletal Class III with open bite is one of the most difficult malocclusion to treat orthodontically. Generally, the morphological characteristics of this malocclusion are poor antero-posterior growth of the maxilla or excessive growth of the mandible, with high angle. ${ }^{7}$ The usual treatment for this type of malocclusion is through the use of auxiliary protraction device, chin cup appliance and surgery.

In this case report, MEAW technique was used which can significantly reduce the treatment time. For patient with open bite condition and dental mid line shift, it is important to restore the balance of occlusal plane (occlusal reconstruction). In 9 month of treatment period, a stable occlusion was obtained, anterior cross bite and open bite was corrected. Dental midline coincided with facial midline.

The use of Multiloop Edgewise Archwire enhance intruding and uprighting of molars. Loops are useful in increasing the range and reducing the force on teeth that are subject to force generated by loops; teeth remote from the loops become the anchorage unit. The MEAW uses a loop between every tooth so that forces on all the teeth are reduced. ${ }^{6}$

The second order bend placed in the MEAW is comparable to what Tweed enthusiasts achieve with anchorage preparation. The loop does not offer a unique force system, they lighten the force and provide excellent hook for elastics. This dentoalveolar camouflage for Class III occlusion is a useful modality when surgery is not desired. Moreover treatment using MEAW system requires relatively short treatment period than the conventional method.

\section{REFERENCES}

1. Sato S. A treatment approach to malocclusions under the consideration of craniofacial dynamics. Grace Printing Press Inc; 2001.

2. Sato S. Alteration of occlusal plane to posterior discrepancy related to development of malocclusion. Introduction to denture frame analysis. Bull Kanagawa Den Col. 1987; 15:115-123

3. Sato S, Suzuki N, Suzuki Y. Longitudinal study of the cant of the occlusal plane and the denture frame in cases with congenitally missing third molars. Further evidence for the occlusal plane change related to the posterior discrepancy. J Jap Ortho Soc. 1988, 47:517-25.

4. Sato S, Suzuki Y. Relationship between the development of skeletal mesio-occlusion and posterior tooth-to-denture base discrepancy. Its significance in orthodontic correction of skeletal Class III malocclusion. J Jap Orthod Soc. 1988; 48:796-810.

5. Sato S, Takamoto K, Suzuki Y. Posterior discrepancy and development of skeletal class III malocclusion. Ortho Rev. 1988 Nov/Dec: $16-29$.

6. Sato S. Developmental characterization of skeletal Class III malocclusion. Angle Orthod. 1994; 64(2):105-11.

7. Sato S, Akimoto S, Matsumato A, Shirasu A, Yoshida J. Chapter Five MEAW Manual. Kanagawa Dental College, Orthodontic Department; autumn 2001.

8. Kim YH. Anterior openbite and its treatment with multiloop edgewise archwire techniques. Angle Orthod. 1987; 57:290-321. 